\title{
Improving Heat Transfer at the Bottom of Vials for Consistent Freeze Drying with Unidirectional Structured Ice
}

\author{
Mónica Rosa, ${ }^{1}$ João M. Tiago, ${ }^{1}$ Satish K. Singh, ${ }^{2}$ Vítor Geraldes, ${ }^{3}$ and Miguel A. Rodrigues ${ }^{1,4}$
}

Received 12 May 2015; accepted 19 October 2015; published online 26 October 2015

\begin{abstract}
The quality of lyophilized products is dependent of the ice structure formed during the freezing step. Herein, we evaluate the importance of the air gap at the bottom of lyophilization vials for consistent nucleation, ice structure, and cake appearance. The bottom of lyophilization vials was modified by attaching a rectified aluminum disc with an adhesive material. Freezing was studied for normal and converted vials, with different volumes of solution, varying initial solution temperature (from $5^{\circ} \mathrm{C}$ to $20^{\circ} \mathrm{C}$ ) and shelf temperature (from $-20^{\circ} \mathrm{C}$ to $-40^{\circ} \mathrm{C}$ ). The impact of the air gap on the overall heat transfer was interpreted with the assistance of a computational fluid dynamics model. Converted vials caused nucleation at the bottom and decreased the nucleation time up to one order of magnitude. The formation of ice crystals unidirectionally structured from bottom to top lead to a honeycomb-structured cake after lyophilization of a solution with $4 \%$ mannitol. The primary drying time was reduced by approximately $35 \%$. Converted vials that were frozen radially instead of bottom-up showed similar improvements compared with normal vials but very poor cake quality. Overall, the curvature of the bottom of glass vials presents a considerable threat to consistency by delaying nucleation and causing radial ice growth. Rectifying the vials bottom with an adhesive material revealed to be a relatively simple alternative to overcome this inconsistency.
\end{abstract}

KEY WORDS: consistency; freeze drying; heat transfer; ice nucleation; unidirectional freezing.

\section{INTRODUCTION}

Freeze drying is widely used in the pharmaceutical and biotechnology industries to improve the stability and longterm storage of protein drugs. However, lyophilization is an expensive process with long processing times and hence the reduction of lyophilization cycle time is an area of focus in industry (1).

Optimization of lyophilization efficiency, reducing the cycle time without compromising product quality, requires good control of heat transfer. Heat transfer from the source (shelf) to the sublimation interface is an important ratelimiting step for primary drying and therefore has received much attention $(2,3)$.

Electronic supplementary material The online version of this article (doi:10.1208/s12249-015-0437-3) contains supplementary material, which is available to authorized users.

\footnotetext{
${ }^{1}$ Centro de Química Estrutural, Department of Chemical Engineering, Instituto Superior Técnico, University of Lisbon, Av. Rovisco Pais, 1049-001, Lisboa, Portugal.

${ }^{2}$ Pharmaceutical Research and Development, BioTherapeutics Pharmaceutical Sciences, Pfizer Inc, Chesterfield, Missouri 63017, USA.

${ }^{3}$ Center of Physics and Engineering of Advanced Materials, University of Lisbon, Av. Rovisco Pais, 1049-001, Lisboa, Portugal.

${ }^{4}$ To whom correspondence should be addressed. (e-mail: miguelrodrigues@tecnico.ulisboa.pt)
}

Heat is exchanged mainly by conduction between the glass vials and the stainless steel shelf on which the vials are loaded, but the walls in the freeze dryer and the shelf above the vials also take part in the heat exchange by radiation (2). Thus, heat transfer to the vial is significantly impacted by the lyophilization vial itself. The bottom of a vial generally has considerable curvature, and therefore, a gap exists between the vial and the shelf. The heat resistance of this gap is added to the resistance of the glass at the bottom of the vial $(3,4)$. The resistance to heat transfer can therefore be greatly reduced by improving the thermal conductivity of this intervening space. Several heat-transfer augmentation devices, which fill this gap, have been described. Pikal et al. (4) concluded that molded and tubing vial design features that have a significant influence on heat transfer may be manipulated to provide improved heat transfer and a more efficient lyophilization cycle. Patel et al. (5), showed the feasibility of using an aluminum quilt between the vials and the shelf. Following this work, Yalkowsky et al. (6) proposed a new device for improvement of heat transfer. This device is an aluminum foil bag which contains a small amount of glycerin, on which the vials are placed. In all cases, the temperature difference between the shelf surface and the product at the vial bottom was significantly reduced, and the drying time could be shortened by about one-third through the use of these devices.

Primary drying involves the sublimation of ice, and the rate of water vapor removal depends on the product 
resistance, which, in turn, is a reflection of how the initial solution was frozen (7). The ice crystal morphology determines indirectly the mass and the heat-transfer rates through the dry layer and, consequently, the freezing parameters have a strong influence on the total duration of the primary and secondary sublimation steps (8). Large ice crystals provide less resistance to water vapor flow during sublimation because they leave behind larger pores. Consequently, by increasing pore size, dry layer resistance is decreased which decreases primary drying time.

The ice crystal structure, once formed, cannot be altered if not annealed, because the subsequent primary drying step only removes the ice via a combination of shelf temperature and chamber pressure (8). Annealing is a process step in which samples are maintained at a specified subfreezing temperature for a period of time to allow for complete crystallization of crystalline compounds and to improve inter-vial heterogeneity and drying rates. However, annealing can have unexpected consequences on product quality. For example, some excipients may crystallize, thus failing to stabilize proteins in the dried state, it may change the $\mathrm{pH}$ of the formulation (9), or give mobility to proteins, which should have much faster aggregation below the freezing temperature because of the higher fraction of unfolded conformation $(10,11)$.

The degree of supercooling achieved by a vial can have a significant impact on its drying behavior. The degree of supercooling, defined as the difference between the equilibrium freezing point and the temperature at which the ice crystals first form in the sample, reflects random nucleation and also depends on the solution properties and process conditions $(12,13)$. The degree of supercooling determines the number of ice crystals formed, which, in turn, affects the porosity of the freeze-dried cake (14). High degree of supercooling results in many small crystals and therefore higher resistance to mass transfer and increased primary drying times. Low degree of supercooling is therefore desirable as it reduces resistance to mass transfer, decreases primary drying times, and improves the process reproducibility (12). An early correlation between ice nucleation temperature and primary drying speed was established by Roy and Pikal (15). Direct analysis of ice crystal size and distribution by optical microscopy in a cold chamber was correlated with sublimation rates by Hottot et al. $(8,16)$. They found that larger ice crystals, generated by annealing of ramp-frozen samples, increased sublimation rates, whereas small ice crystals, prepared by liquid nitrogen immersion, resulted in rather low sublimation rates. The link between ice nucleation, temperature, and sublimation rates is therefore considered to be well-understood.

Ice nucleation is a stochastic event, and the probability of spontaneous ice crystal formation is, among others, dependent on the presence of foreign particles. Ice nucleation and, in consequence, ice crystal size distribution will generally differ from vial to vial, resulting in a large sample heterogeneity within one batch (17). Controlling ice nucleation temperature is expected to lead to a more uniform product because the degree of supercooling influences product parameters, for example, cake resistance, specific surface area, and residual moisture.

A number of earlier techniques to control ice nucleation have demonstrated the potential for process improvement. Recently Kasper et al. (17) reviewed techniques like shelf- ramped freezing, precooled shelf method, electro freezing, ultrasound-controlled ice nucleation, quench freezing or directional freezing.

Rambhatla et al. (7) developed the "ice fog" technique, where cold nitrogen gas is introduced into the chamber to form an "ice fog," thereby facilitating nucleation of samples at the temperature of interest. Ultrasound-controlled ice nucleation, published by Hottot et al. (18), was other technique developed for controlling ice nucleation. Recently, a method was developed that involves pressurizing the product chamber with an inert gas up to 2 bar overpressure and a rapid release by evacuation, inducing instantaneous ice nucleation (19). Geildobler et al. (20) also develop a technique for controlling nucleation that involves loading small ice crystals to the chamber by a repressurization step through the venting valve of the compartment of the condenser, which has some water load. More recently Kuu et al. (21) reported that inserting an air space between the vials and the shelf could increase the nucleation temperature, which contributed to increase ice porosity and consequently reduce drying time.

Most of these techniques favor nucleation at the top of the vials, thus causing the first freezing front to progress from top to bottom; after which, the solution will continue freezing from bottom to top. Other nucleation techniques (9) have been proposed which favor nucleation at the bottom and directional freezing, by placing the vial on dry ice followed by slow freezing on a precooled shelf. This approach is more in line with the work developed herein. However, instead of lowering the temperature with dry ice to favor nucleation at the bottom, we fill the curvature gap at the bottom of the vial to enhance its heat transfer on normal lyophilization shelves. Consistent nucleation has been achieved previously by suppressing natural convection in unidirectional freezing (bottom to top) (22). In this work, we evaluate how this freezing geometry can improve the structure of ice for enhancing lyophilization. A computational fluid dynamics (CFD) model was developed to interpret the impact of the air gap on the heat transfer and the advantages and limitations of different freezing geometries and parameters.

\section{MATERIALS AND METHODS}

\section{Materials}

One commonly excipient was used, 4\% ( $w / v)$ mannitol were studied in the lyofilization systems. D-mannitol USP were obtained from Sigma-Aldrich (St. Louis, MO). Mannitol is a fine example of crystalline system that is commonly used as a bulking agent.

Analytical-grade L-histidine base and L-histidine hydrochloride monohydrate were purchased from AppliChem (Gatersleben, Germany). Distilled water, used to make all the solutions, was treated with Milli-Q Integral 3 Pure (Millipore, USA). The buffer used was $10 \mathrm{mM}$ histidine at $\mathrm{pH}=5.5$.

\section{Conversion of the Vials}

Vials (5-mL Rotilabo, Germany) were converted by filling the air gap curvature of the vial base with superconductive epoxy (Omegabond 100, Omega Engineering, USA) that was 
uniformly spread and compressed by a circular and planar aluminum sheet ( $2 \mathrm{~mm}$ thick).

\section{Lyophilization}

Aliquots of $2 \mathrm{~mL}$ were lyophilized in the $5-\mathrm{mL}$ vials (Rotilabo, Germany) using a Christ Alpha 2-4 LSC Freeze Dryer (SciQuip, UK). The setup is schematically described in Fig. 1. In this geometry, the vials are exposed to the condenser at $-85^{\circ} \mathrm{C}$. For this reason, an aluminum shield was used to prevent loss of heat to the condensers by radiation. The vials were placed on a precooled freeze dryer shelf at $-35^{\circ} \mathrm{C}$ and held at this temperature for 6 or $3 \mathrm{~h}$ before initiating drying. Primary drying proceeded at 0.1 mbar vacuum with the shelf temperature set at $-10^{\circ} \mathrm{C}$, for $20.5 \mathrm{~h}$. Secondary drying consisted of two steps; $4 \mathrm{~h}$ to reach $25^{\circ} \mathrm{C}$ and $11 \mathrm{~h}$ at $25^{\circ} \mathrm{C}$, 0.08 mbar. An aggressive cycle was performed, where primary drying was carried at 0.1 mbar with the shelf temperature set at $-5^{\circ} \mathrm{C}$, for $14 \mathrm{~h}$. The secondary drying was the same in both cycles.

To understand the contribution of the ice structure on the primary drying enhancement, regardless of the heat-transfer acceleration provided by flat base, converted vials were also frozen radially by insulating the bottom during freezing. This insulator (polystyrene with a thickness of $3 \mathrm{~cm}$ ) was removed before the drying.

Temperature profiles were obtained by inserting a temperature probe at the center of the vials. Triplicate temperature profiles were obtained for each vial type (normal or converted) by running triplicate lyophilization runs (one temperature profile was measured per run).

\section{Scanning Electron Microscopy}

The lyophilized cake structures were examined using a scanning electron microscope (SEM; JEOL JSM-7001F EDS Oxford INCA 250). Particle samples were coated prior to measurement with a gold film by electrodeposition in vacuum. Acceleration voltage during observation was $25 \mathrm{kV}$.

\section{Differential Scanning Calorimetry}

Thermal Analysis (by differential scanning calorimetry (DSC)) was performed using a Thermal Advantage DSC Q1000 V9.8 (TA Instruments-Waters, LLC) that was calibrated for temperature and cell constants using indium and sapphire. Samples $(10 \mu \mathrm{L})$ were crimped in non-hermetic aluminum pans $(30 \mu \mathrm{L})$. The sample is quickly cooled to a temperature of $-65^{\circ} \mathrm{C}$, held for $30 \mathrm{~min}$, and then heated at a rate of $5^{\circ} \mathrm{C} / \mathrm{min}$ back to room temperature under a continuously purged dry nitrogen atmosphere (flow rate of $50 \mathrm{~mL} /$ $\mathrm{min})$. The glass transition temperatures $\left(\mathrm{Tg}^{\prime}\right)$ of the frozen solutions were obtained from the DSC thermograms (23).

\section{Unidirectional Freezing Equipment}

The unidirectional freezing setup used in this work to simulate freezing in a lyophilization system (lyo-UFS) is represented in Fig. 2. This setup is composed of a thin block with a circular heat-transfer surface with $45 \mathrm{~mm}$ of diameter and a cavity for internal circulation of a cryogenic fluid (ethanol $96 \%, v / v)$. The temperature was controlled by a Julabo (Japan) cryostat model R CF-41. The heat-transfer surface was delimited by an acrylic cover with $15 \mathrm{~mm}$ walls and $50 \mathrm{~mm}$ of height. Three lyophilization vials were placed over the heat-transfer surface, obtaining triplicate results in each experimental assay. The front and top views of lyo-UFS are represented in Fig. 2a, b, respectively.

\section{Determination of the Nucleation Time}

Lyophilization vials containing a solution of $4 \%$ mannitol in histidine buffer $10 \mathrm{mM}$ ( $\mathrm{pH}$ 5.5) were frozen in the lyo-UFS. A video camera was used to record the nucleation phenomenon in each vial and determine the nucleation time.

Two classes of vials were tested: normal glass vials and glass vials with the base rectified with superconductive epoxy (converted vials). With each type of vials, three parameters were tested: initial solution temperature $\left(5^{\circ} \mathrm{C}\right.$ and $\left.20^{\circ} \mathrm{C}\right)$, solution height $(1$ and $3 \mathrm{~cm})$, and the heat-transfer surface temperature $\left(-35^{\circ} \mathrm{C}\right.$ and $\left.-20^{\circ} \mathrm{C}\right)$.

\section{Determination of the Reconstitution Time}

Water was added to the lyophilized cake to reconstitute the initial concentrations. The sample was held without agitation until a clear solution was observed; the time required was recorded.

\section{Powder X-ray Diffraction}

The XRD patterns for the samples in this work were collected using two different X-ray powder diffractometers: a D8 Advance Bruker AXS $\theta-2 \theta$ diffractometer, with copper

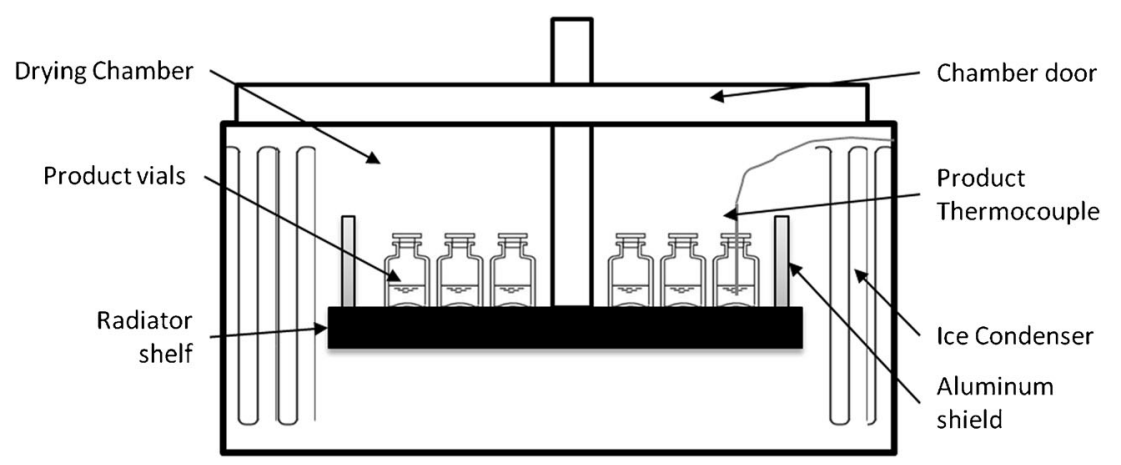

Fig. 1. Schematic diagram of the freeze dryer system 


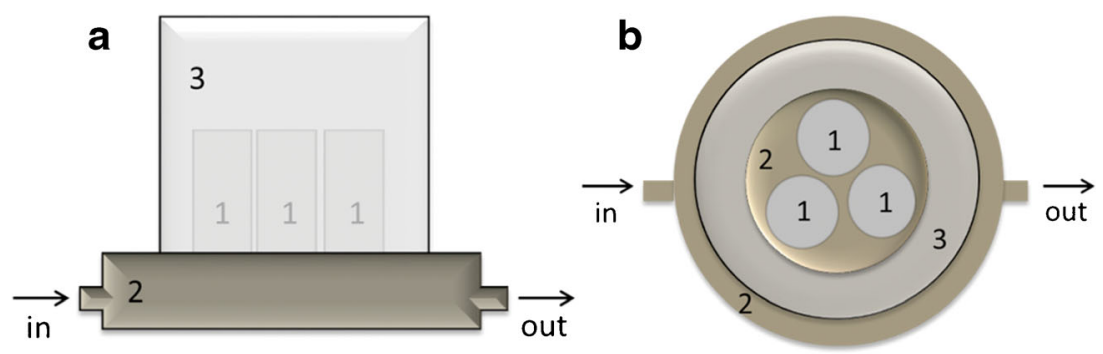

Fig. 2. Schematic representation of the device used for unidirectional freezing of lyophilization vials, lyo-UFS: front view (a) and top view (b). The lyo-UFS system is formed by three lyophilization vials (1), a heat-transfer surface (2), and an acrylic cylinder cap (3). In and out flow direction of cryogenic fluid

radiation $(\mathrm{Cu} \mathrm{K} \alpha, \lambda=1.5406 \AA)$ and a secondary monochromator.

The tube voltage and current were $40 \mathrm{kV}$ and $40 \mathrm{~mA}$, respectively. The divergence slit and anti-scattering slit settings were variable for illumination of the $20-\mathrm{mm}$ sample. Samples were initially scanned between $5^{\circ}$ and $35^{\circ} 2 \theta$ in aluminum sample holders.

\section{Computational Fluid Dynamics Model}

The unsteady-state distribution of temperature of a liquid inside the vial, with or without natural convection, was predicted by solving the continuity, momentum, and energy equations in that domain, given by

$$
\frac{\partial \rho}{\partial t}+\nabla \cdot \mathrm{U}=0
$$

$$
\rho \frac{\partial \mathrm{U}}{\partial t}+(\mathrm{U} \cdot \nabla) \mathrm{U}=-\nabla p+\mu \nabla^{2} \mathrm{U}+\rho \mathrm{g}
$$

$\rho C_{p} \frac{D T}{D t}-\nabla \cdot k \nabla T=0$

where $\mathbf{U}$ is the velocity vector field, $\mathbf{g}$ is the gravity acceleration vector, $p$ and $T$ are the pressure and temperatures fields respectively, and $\rho, \mu, C_{\mathrm{p}}$, and $k$ are the density, dynamic viscosity, heat capacity, and thermal conductivity of the fluid, respectively. The temperature distribution inside the vial's walls was determined by solving the energy equation in that domain using the physical properties of the material. The physical properties used in simulation are displayed in Table I.

The governing differential equations were solved using a solver developed elsewhere (22), based on the open-source package OpenFOAM (version 2.3.0) (24). The transport equations were discretized by the finite volume method applied to arbitrary-shaped cells, using a segregated approach and collocated variables. The transport equations were discretized with a fourth-order-accurate scheme in space and second-order-accurate scheme in time. The coupling between equations and in particular the pressure-velocity coupling was performed by the hybrid SIMPLE-PISO algorithm with three outer correctors (24).

A two-dimensional (2D) structured mesh of $120 \times 50$ cells, with half of the nodes in the liquid domain, was sufficient to ensure grid-independent results. The results were postprocessed with the open-source program Paraview (25).

To estimate a reasonable range for the nucleation time, simulations were performed for the extreme temperatures of a typical range of nucleation temperatures observed for sucrose solutions $\left(-13^{\circ} \mathrm{C}\right.$ and $\left.-18^{\circ} \mathrm{C}\right)(13)$. The simulations were run with a time step of $0.01 \mathrm{~s}$ until the nucleation temperature was reached in some part of the fluid mixture.

The initial temperature of the vials was assumed uniform. For the boundary conditions, we assumed that the temperature in the vial's base was uniform and that the lateral and top walls were adiabatic.

Two 2D geometries were studied; normal vials with a concave bottom (used in experimental runs) and perfectly flat bottom "ideal" vials. Physical properties of the materials of the vial are described in Fig. 3.

\section{RESULTS}

Figure 4 represents the experimental and simulated nucleation time for normal vials and converted vials under various freezing conditions. Approximately 150 freezing runs were carried and nucleation time measured. The nucleation event was distinguished in the recorded videos by the formation of an ice layer at the bottom of the vials or in other cases as the formation of a cloud that rapidly covered the liquid phase (most frequently for the normal vials). The experimental values are represented in Fig. 4 by red and green dots, for normal and converted vials, respectively. The nucleation time in converted vials (green dots) is considerably more reproducible and therefore difficult to distinguish in Fig. 4; for this reason, all the experimental values are also presented in the Annex (Table A1). The averages of the observed nucleation times are shown in Fig. 4 (large empty symbols), which can be easily compared with the values obtained by CFD simulation; the average experimental values are also shown in Table II. Nucleation times were calculated for both limits of the nucleation temperature range $\left(-13^{\circ} \mathrm{C}\right.$ and $\left.-18^{\circ} \mathrm{C}\right)$. The average value is shown in Fig. 4 for normal and converted vials under different conditions, with corresponding standard deviation bars. The simulations correlate well with the experimental data, especially for the converted vials which also show consistently shorter nucleation time. Overall, Fig. 4 shows that the 
Table I. Physical Properties of the Fluid (Aqueous Solution with 4\% (w/w) Mannitol) and Materials of the Vial

\begin{tabular}{lllrr}
\hline & \multicolumn{1}{c}{ Density $\left(\mathrm{kg} / \mathrm{m}^{3}\right)$} & Viscosity $(\mathrm{Pa} \mathrm{s})$ & $\mathrm{C}_{\mathrm{P}}\left(\mathrm{J} \mathrm{kg}^{-1} \mathrm{~K}^{-1}\right)$ & ${\mathrm{Thermal} \mathrm{conductivity}\left(\mathrm{W} \mathrm{K}^{-1} \mathrm{~m}^{-1}\right)}$ \\
\hline Solution & $1001.5-0.0191 \times(T-273.15)$ & $1.85 \times 10^{-3} e^{-8.19}\left(\frac{T}{273.15}-1\right)$ & 3950 & 0.55 \\
Glass & 2600 & - & 840 & 1.05 \\
Air & 1.2 & - & 1000 & 0.024 \\
Epoxy & 1000 & - & 890 & 7.2 \\
Silicone & 2300 & - & 705 & 0.26 \\
PVC & 1200 & - & 1670 & 0.19 \\
\hline
\end{tabular}

nucleation time increases with liquid height and decreases with shelf temperature. The simulations suggest that liquid height has particular impact on the nucleation consistency in normal vials. This effect results from the larger quantity of liquid, which also increases the time required for the solution to be cooled from $-13^{\circ} \mathrm{C}$ to $-18^{\circ} \mathrm{C}$, therefore increasing the time span wherein nucleation can happen.

The normal vials present an edge curvature and a convex surface, in the base (Fig. 3a). The simulation (Fig. 5a) shows that in these vials, a significant part of the liquid reaches freezing temperature (below $0^{\circ} \mathrm{C}$ ) before nucleation temperature is achieved, which is defined by the isothermal (dotted) line at $0^{\circ} \mathrm{C}$ marked on Fig. 5a. The temperature gradient is therefore not very steep, and it takes a long time for any liquid domain to reach the nucleation temperature range. However, the simulation of the converted vial reveals that a layer of solution cools much faster at the bottom (e.g., comparing $15 \mathrm{~s}$ instance of Fig. 5a, b temperature histograms), rapidly reaching the nucleation temperature in this layer, while the rest of the solution remains close to the initial temperature. Furthermore, the cooling in the converted vial is similar to the ideal vial, except at the vial's edge, which shows slight influence in the temperature distribution.

Table III shows the calculated time to reach the extremes of the assumed nucleation temperature interval if different adhesive materials were used to fill the bottom curvature gap. Table III also shows that the heat-transfer coefficient from the vial's bottom increases almost three times by replacing the air with the superconductive epoxy.

The experimental freeze-drying results revealed that the converted vials have a lower primary drying time (Fig. 6; Table IV). For comparative purposes, we considered the time period measured from the vacuum pump start (after the plateau at freezing temperature) until the product reached $1^{\circ} \mathrm{C}$ less than the temperature set for the shelf, because the normal vials never reached the shelf temperature, even when primary drying time was substantially extended. The primary drying time (Table IV) for normal vials was approximately $805 \mathrm{~min}$ when shelf temperature was $-10^{\circ} \mathrm{C}$ and 500 min when the shelf temperature was $-5^{\circ} \mathrm{C}$. The converted vials enabled a reduction of the primary drying time in approximately $280 \mathrm{~min}$ $(35 \%)$ when the shelf was set to $-10^{\circ} \mathrm{C}$ and $125 \mathrm{~min}$ $(25 \%)$ when the shelf temperature was $-5 \mathrm{C}$.

The individual temperature profiles measured for the normal cycle are almost coincident for both vial types as observed in Fig. 6c, e. Some variation is observed in the temperature profiles of the aggressive cycle (Fig. 6d, f).

The SEM images show different morphology between samples lyophilized in converted or normal vials (Fig. 7). In converted vials, the cake revealed a structured honeycomb appearance with large interconnected pores.
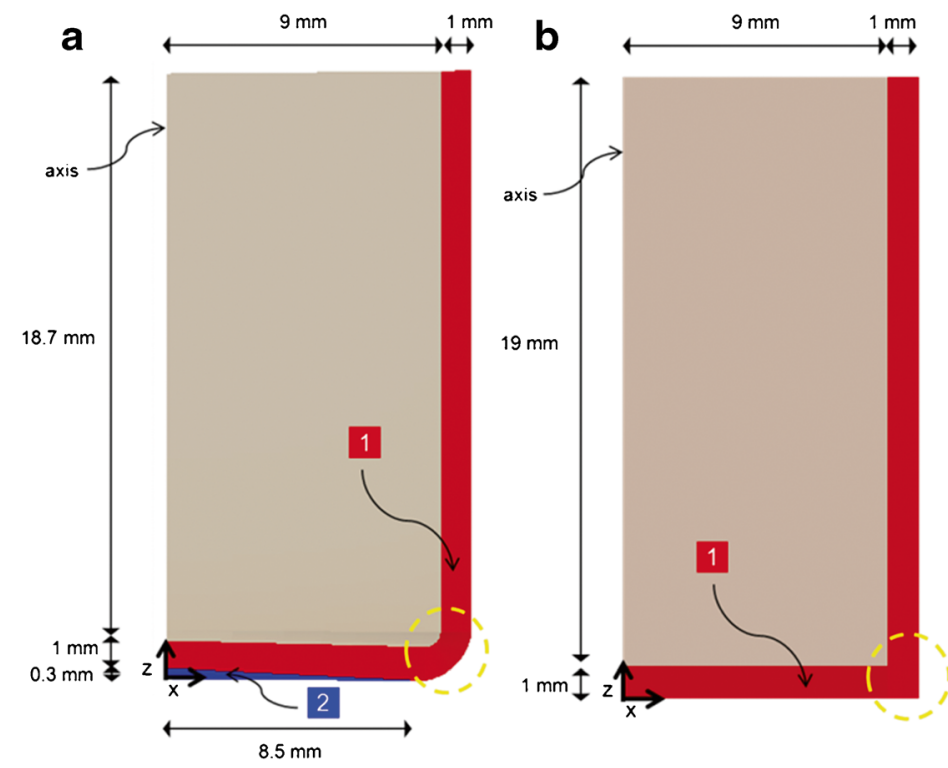

\section{1 glass wall \\ $\rho=2600 \mathrm{~kg} / \mathrm{m}^{-3}$ \\ $c_{p}=840 \mathrm{~J} \mathrm{~kg}^{-1} \mathrm{~K}^{-1}$ \\ $\mathrm{k}=1 \mathrm{Wm}^{-1} \mathrm{~K}^{-1}$}

2 a) conductive glue

$\rho=1000 \mathrm{~kg} / \mathrm{m}^{-3}$

$c_{p}=1000 \mathrm{~J} \mathrm{~kg}^{-1} \mathrm{~K}^{-1}$

$\mathrm{k}=7.2 \mathrm{Wm}^{-1} \mathrm{~K}^{-1}$

b) air

$\rho=1.2 \mathrm{~kg} / \mathrm{m}^{-3}$

$c_{p}=1000 \mathrm{~J} \mathrm{~kg}^{-1} \mathrm{~K}^{-1}$

$\mathrm{k}=0,024 \mathrm{Wm}^{-1} \mathrm{~K}^{-1}$

Fig. 3. Computational domain and physical properties of normal/converted vial (a) and ideal vials with maximum heat-transfer bottom (b). The dashed circles highlight the main difference between referred vials.

Density, specific heat capacity, and thermal conductivity are represented by $\rho, C_{\mathrm{p}}$, and $k$, respectively 

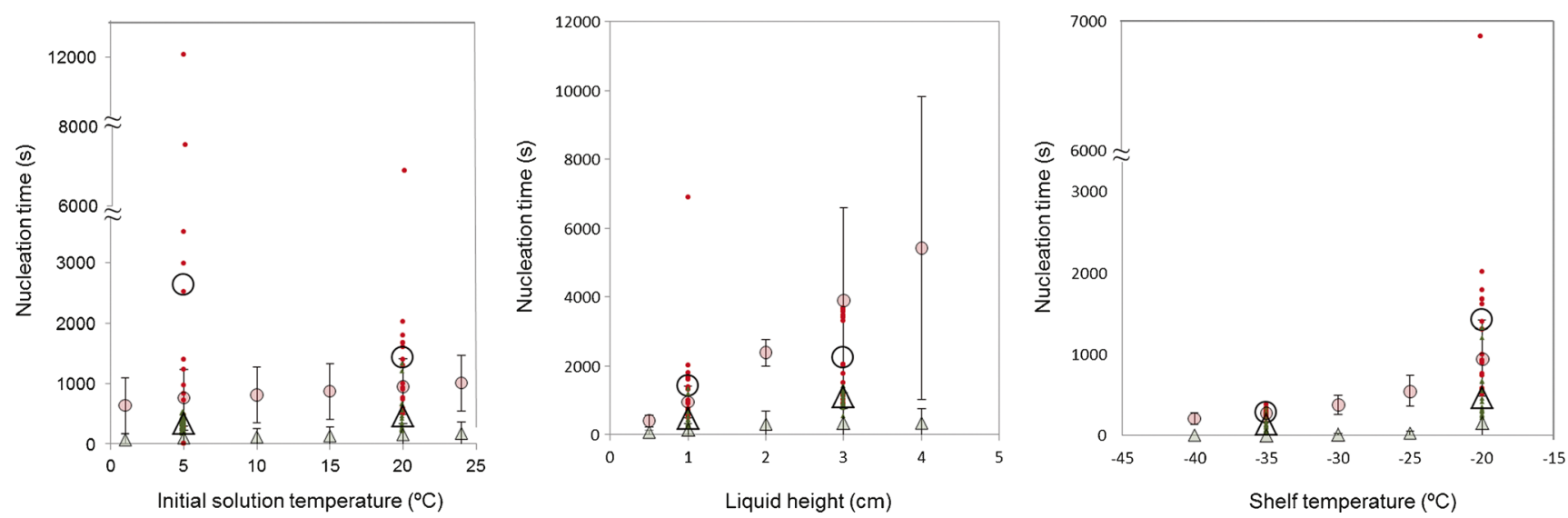

Fig. 4. Impact of different parameters on the nucleation time of $4 \%$ mannitol solutions in histidine buffer for normal and converted vials: a initial solution temperature (for $1 \mathrm{~cm}$ of liquid height and $-20^{\circ} \mathrm{C}$ shelf temperature); b solution height (for $20^{\circ} \mathrm{C}$ of initial solution temperature and $-20^{\circ} \mathrm{C}$ shelf temperature); $\mathbf{c}$ shelf temperature (for $20^{\circ} \mathrm{C}$ of initial solution temperature and $1 \mathrm{~cm}$ of liquid height). Experimental values are represented by red dots for normal vials and green dots for converted vials; the corresponding averages are represented by empty circles and empty triangles; and the nucleation times represented by pink circles and green triangles are an average of the simulated times (for nucleation at $-13^{\circ} \mathrm{C}$ and $-18^{\circ} \mathrm{C}$ ), for normal vials and converted vials

Independently of the heat-transfer resistance at the bottom of the vials, the structure and orientation of ice crystals may also constitute an important limitation. Figure 8 shows the temperature profiles obtained during the lyophilization of solutions using converted vials that were frozen in distinct directions (radial and axial); normal vials (normally lyophilized) were also used as control (dotted line). The drying time required for radially frozen vials, was similar to unidirectional frozen vials (Fig. 8), but the cake aspect was distinct (Fig. 9). In the normal vial, we observed a small peak at the center, while in converted vials frozen from bottom to top, the cake shows no peak, a vertically oriented structure is observed. A rough and non-uniform appearance of the cake was observed when the converted vials were frozen radially; moreover, apparent mannitol's crystals were observed. Figure 10 shows comparative powder X-ray diffraction (PXRD) diffractograms of the raw mannitol (a) and the freeze-dried cakes. Distinctive diffraction peaks can be observed for both normal and converted vials, suggesting the presence of $\delta$-mannitol polymorph. This crystalline form has been previously attributed to fastfreezing rates during lyophilization (26). The powders obtained from converted vials that were radially frozen show the characteristic peaks of raw mannitol (arrows), perhaps due to the slower freezing or some recrystallization.
The glass transition temperature of the solution determined by DSC was $-37.9 \pm 0.06^{\circ} \mathrm{C}$.

No measurable difference was observed in reconstitution time regardless of the vials used. The reconstitution time was approximately $20 \mathrm{~min}$ in all the cases.

\section{DISCUSSION}

The results in this study provide a demonstration of the impact of the bottom curvature of vials on the freeze-drying process, which is herein emphasized by the improvement on nucleation, ice structure orientation, and primary drying time, when this curvature was rectified with an adhesive material. Figures 4 and 5 and Table III show the improvement promoted by the converted vials, which was confirmed experimentally and by the simulations. The bottom of a normal vial has considerable curvature, and therefore, an air gap exists between the vial and the shelf. The experimental results shown in Fig. 4 emphasize the limitation caused by air, because when this gap was filled with the conductive epoxy (converted vials), the nucleation time was reduced several times, being the highest difference (eight times) observed for the highest shelf temperature $\left(-20^{\circ} \mathrm{C}\right)$. This can be explained by the much higher thermal conductivity of solid materials compared with

Table II. Average Nucleation Time Observed in Lyophilization Vials Containing Solutions of Mannitol (4\% Mass) in Histidine Buffer Under Different Conditions: Initial Solution Temperature, Liquid Height, Shelf Temperature, and Vial Type (Normal and Converted)

\begin{tabular}{|c|c|c|c|c|c|}
\hline Initial temperature $\left({ }^{\circ} \mathrm{C}\right)$ & Liquid height $(\mathrm{cm})$ & Shelf $T\left({ }^{\circ} \mathrm{C}\right)$ & Vial type & Average nucleation time (s) & $\mathrm{SD}(\mathrm{s})$ \\
\hline \multirow[t]{4}{*}{5} & 1 & -20 & Normal & 2628 & 3260 \\
\hline & & & Converted & 334 & 98 \\
\hline & 3 & -20 & Normal & 2250 & 1120 \\
\hline & & & Converted & 1096 & 2467 \\
\hline \multirow[t]{4}{*}{20} & 1 & -20 & Normal & 1425 & 1449 \\
\hline & & & Converted & 464 & 325 \\
\hline & & -35 & Normal & 272 & 47 \\
\hline & & & Converted & 141 & 92 \\
\hline
\end{tabular}


a

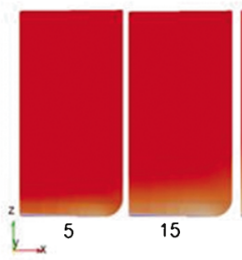

b

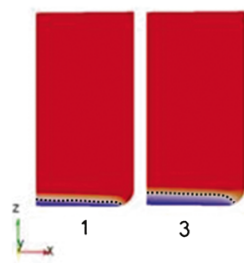

C

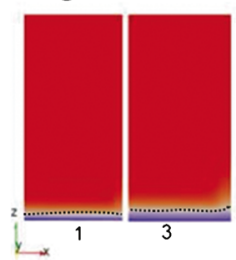

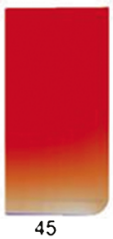

45

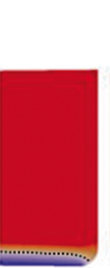

6
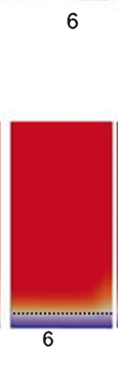

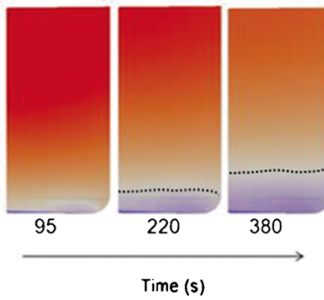

Time (s)

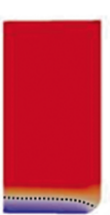

9
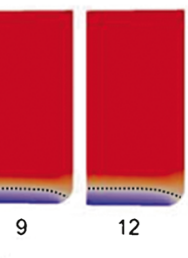

12

Time (s)
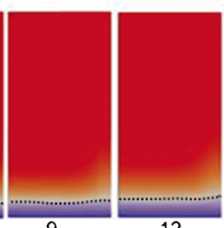

...

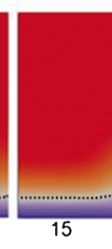

Time (s)
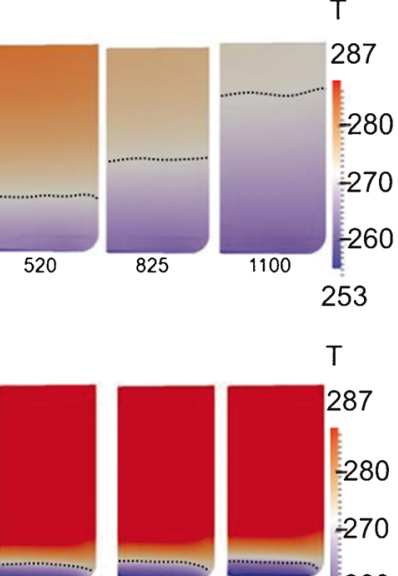

20
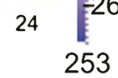

$\mathrm{T}$

$\top$
287
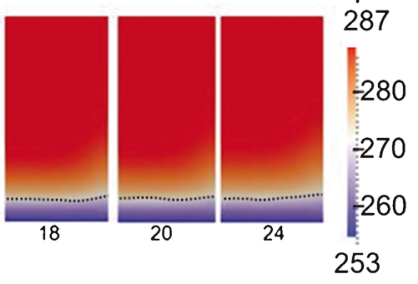

Fig. 5. Temperature histogram predicted by CFD model for a normal lyophilization vial (a), a converted (rectified epoxy base) vial (b), and an ideal vial (c). The simulated parameters were $20^{\circ} \mathrm{C}$ of initial solution temperature, $-20^{\circ} \mathrm{C}$ of shelf temperature, and $2 \mathrm{~cm}$ of liquid height. Dashed lines represent points at same temperature ( $T=273.15 \mathrm{~K})$

air; for this reason, any adhesive material or polymer can provide equivalent results, as the simulations suggest (Table III). In this case, replacing the air by the conductive epoxy should increase the heat transferred through the bottom three times.

In normal vials, nucleation causes the formation of a "cloud" of ice that covers the entire liquid domain, which correlates well with the simulated temperature histogram shown in Fig. 5a. When nucleation happens, all the liquid in the vial is below freezing temperature, causing ice crystals to grow rapidly throughout the entire liquid domain, i.e., all the domain underneath the $0^{\circ} \mathrm{C}$ isothermal line shown on Fig. 5a. In the converted vials, the nucleation site was consistently observed at the edge of the bottom surface. Nucleation was in this case followed by the formation of a thin layer of ice at the bottom. Both lower shelf temperature and lower volume of liquid contribute for faster cooling of the base. Under bottom to top freezing, natural convection is significantly suppressed and should have little influence on heat transfer (22); therefore, the liquid cooling is essentially determined by conduction across the vial's base and thermal diffusion from the liquid's bottom interface to the bulk. The critical aspect for reproducible nucleation at the bottom surface relies therefore on enabling a cooling rate that can substantially overcome heat thermal diffusion, otherwise most of the liquid goes below $0^{\circ} \mathrm{C}$ before the bottom layer reaches the nucleation temperature. Our results show that lower shelf temperature minimizes the impact of the bottom heat resistance, as expected because of the higher temperature differential. However, this is not easily achieved in industrial systems with shelves

Table III. Nucleation Time Simulated by CFD Model for Using Various Materials at the Vial's Bottom and Average Heat-Transfer Coefficient, Considering $1 \mathrm{~cm}$ of Solution Height, $20^{\circ} \mathrm{C}$ of Initial Solution Temperature, and $-20^{\circ} \mathrm{C}$ for Shelf Temperature

\begin{tabular}{lccc}
\hline Material at the base (Fig. 2a) & Heat-transfer coefficient (bottom) & Time (s) to reach nucleation temperature of: \\
\cline { 3 - 4 } & $\mathrm{Jm}^{2} \mathrm{~K}^{-1}$ & $-18^{\circ} \mathrm{C}$ & $-13^{\circ} \mathrm{C}$ \\
\hline Normal (air gap) & 341 & 1281 & 626 \\
PVC & 717 & 519 & 151 \\
Silicone & 768 & 280 & 49 \\
Epoxy & 987 & 227 & 34 \\
Ideal vial (Fig. 2b) & 1000 & 26
\end{tabular}

${ }^{a}$ The average heat-transfer coefficient was calculated by simulating heat-transfer in steady state across the bottom of the vial, using a constant temperature difference (area-average heat flux divided by the temperature difference) 

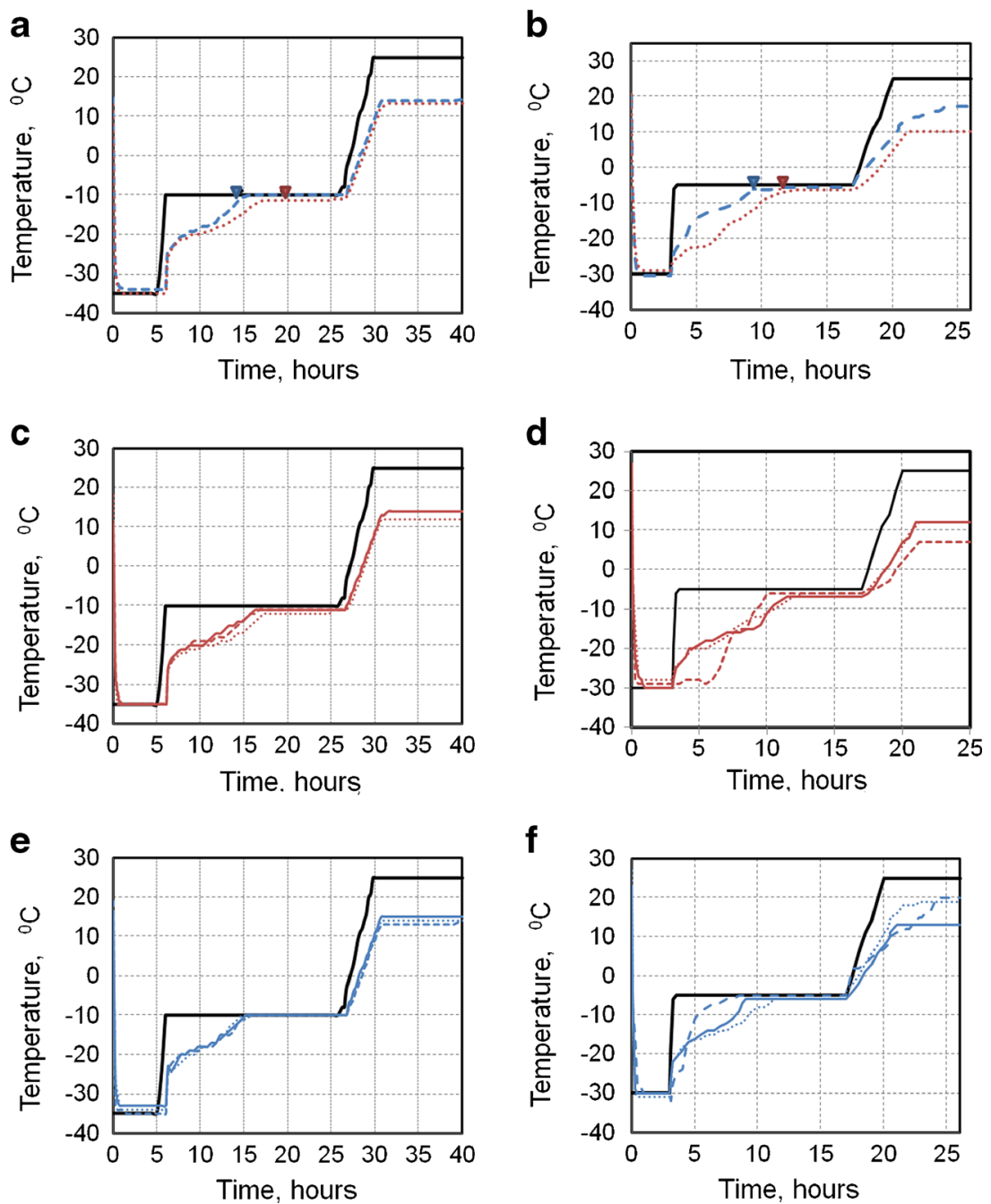

Fig. 6. Temperature profiles obtained during the lyophilization of solutions containing $4 \%$ mannitol in $10 \mathrm{mM}$ His- $\mathrm{HCl}$ ( $\mathrm{pH} 5.5$ ); shelf temperature is represented by the black line. a Average of three runs under normal cycle for normal vials (dotted red line) and converted vials (dashed blue line). b Average of three runs under aggressive cycle for normal vials (dotted red line) and converted vials (dashed blue line). c Three individual runs under normal cycle in normal vials; $\mathbf{d}$ three individual runs under aggressive cycle in normal vials; e three individual runs under normal cycle in converted vials; $\mathbf{f}$ three individual runs under aggressive cycle in converted vials. The end of primary drying for normal and converted vials is highlighted by triangular marks

containing thousands of vials; cooling ramps of few degrees Celsius per minute are typical. Nonetheless, our results are evidence that regardless of the cooling method, the poor conductivity caused by air always presents a serious limitation for inducing nucleation at the bottom surface.

Table IV. Primary Drying Time for Normal Vials and Converted Vials Under Normal Cycle and Aggressive Cycle

\begin{tabular}{lcc}
\hline & $\begin{array}{l}\text { Primary drying time for } \\
\text { normal cycle (min) }\end{array}$ & $\begin{array}{l}\text { Primary drying time for } \\
\text { Aggressive cycle (min) }\end{array}$ \\
\hline $\begin{array}{l}\text { Normal } \\
\text { vial }\end{array}$ & 805 & 500 \\
$\begin{array}{l}\text { Converted } \\
\text { vial }\end{array}$ & 525 & 375 \\
\hline
\end{tabular}

The simulations were generally optimistic regarding the prediction of the nucleation time. This can be explained by the use of a high range for the nucleation temperature $\left(-18^{\circ} \mathrm{C}\right.$ to $-13^{\circ} \mathrm{C}$ ) or most likely due to an imperfect heat conduction between the several interfaces, which is not considered in the model.

A significant decrease in primary drying time (35\%) was also obtained with the converted vials due to better heat conduction-comparable with what was obtained in other study (6) using a fluid cushion device. Several studies have shown that the primary drying time can be reduced by enhancing the heat transfer from the bottom. The air layer at the vial's bottom is particularly limiting heat transfer for lower temperature differential. The nucleation time and primary drying time in normal and converted vials should converge for increasing temperature differential. Therefore, one 

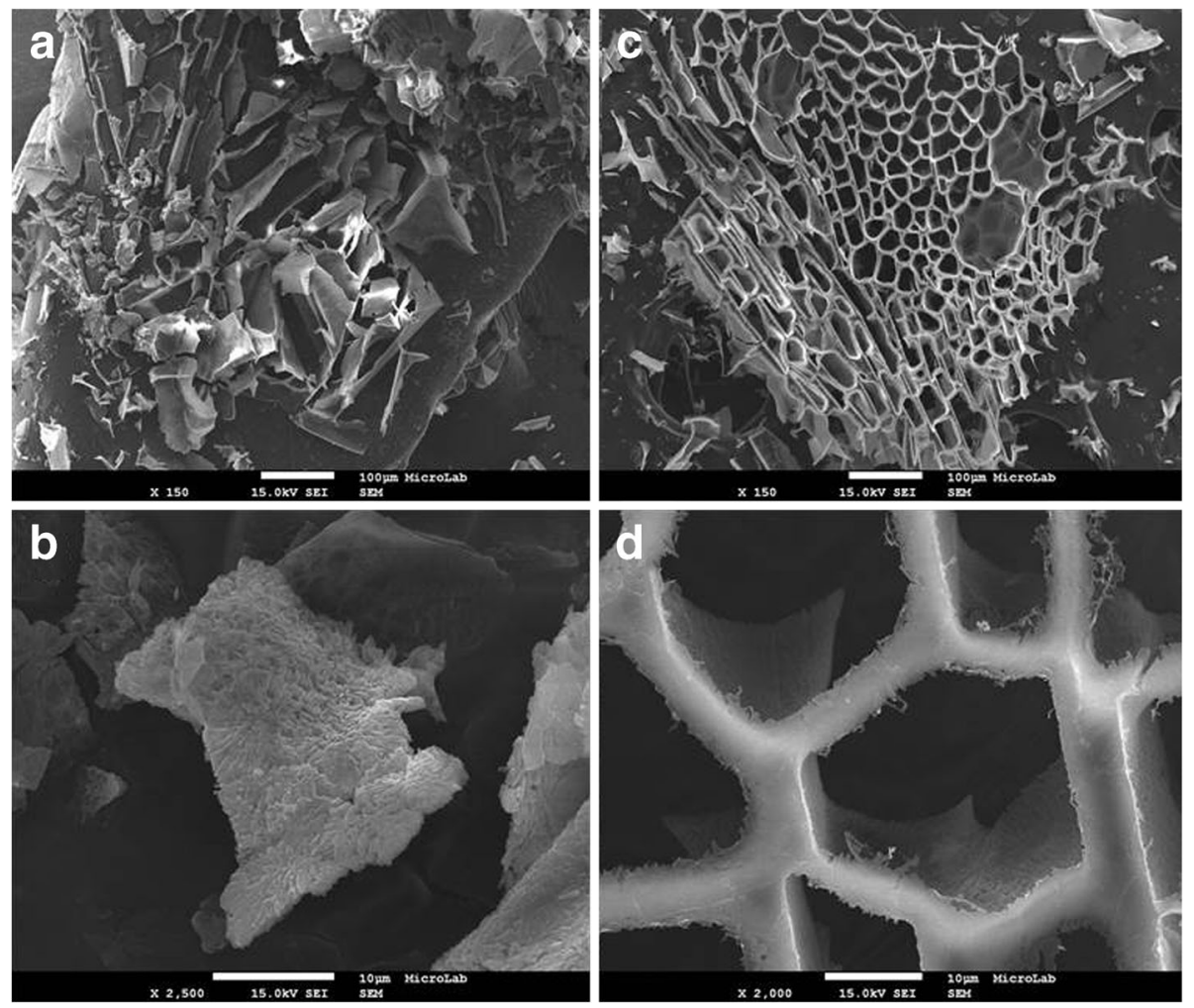

Fig. 7. SEM images of selected cakes in normal vials $(\mathbf{a}, \mathbf{b})$ and in converted vials $(\mathbf{c}, \mathbf{d})$

obvious alternative for overcoming the air barrier limitation to primary drying is to increase shelf temperature. However, high heat-transfer rates during primary drying are not always convenient because the vapor pressure in the cake may increase due to the high sublimation rate, leading to its collapse and poor appearance. This is particularly relevant when the shelf temperature is above the solution $\mathrm{T}_{\mathrm{g}}{ }^{\prime}$ (in our case $-37.9^{\circ} \mathrm{C}$ ). This helps to explain the appearance of the cake in the converted vial that was frozen radially before lyophilization (in Fig. 9a). The cake appearance was rough, and small fragments can be seen all over its surface, which correlates well with the different mannitol polymorph observed by PXRD (Fig. 10). Conversely, the cake appearance of the unidirectionally frozen vials was very good, even though the vial was converted and drying conditions were the same as those radially frozen. This result evidences the importance of the ice structure, compared with the influence of better heat conduction per se. The microscopic honeycomb structure of the final cake (Fig. 7) clearly illustrates the reason for the better lyophilization performance. Unidirectional freezing created large dendritic ice crystals, which could be observed in the cake as large and unidirectional pores (Fig. 7). The unidirectional geometry allows high sublimation rates without increasing the internal cake pressure, due to the connected and continuous nature of the cake pores. During the sublimation, since the dried-product layer is more structured, there is less resistance to water removal favoring the cake uniformity. Interestingly, Kuu et al. (21) have reached an equivalent result
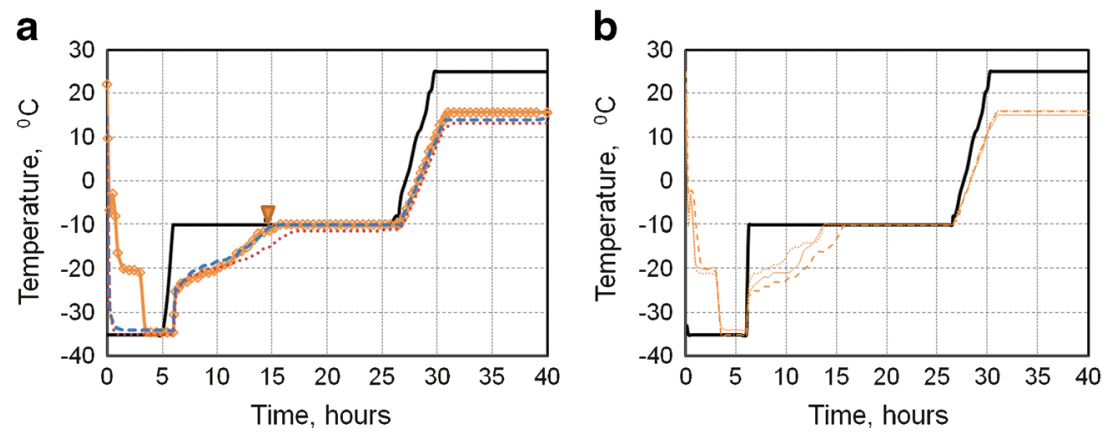

Fig. 8. Temperature profiles obtained during the lyophilization of solutions containing $4 \%$ manitol in $10 \mathrm{mM}$ His- $\mathrm{HCl}$ ( $\mathrm{pH}$ 5.5) under normal cycle; shelf temperature is represented by the black line. a Average of three runs in normal vials (dotted red line); converted vials frozen from bottom to top (dashed blue line); and converted vials frozen radially (orange squares with line). b Three individual runs in converted vials frozen radially; other corresponding individual plots are presented in Fig. 6 


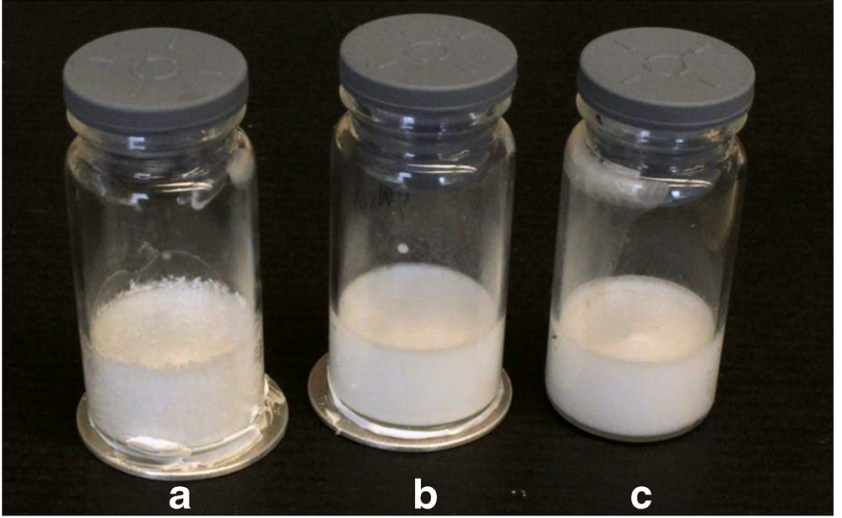

Fig. 9. Aspect of lyophilized cake in converted vials with radial freezing (a), converted vials with unidirectional freezing (b), and normal vials (c)

(33\% improvement in primary drying for corresponding concentration) by following an apparently opposite strategy, i.e., by increasing the air gap between the shelf and the vials. Nonetheless, both approaches improved ice porosity. Herein, the temperature gradient enables ice crystals to grow into a hot liquid phase, favoring the formation of large columnar dendrites, as also inferred from the SEM images. Kuu et al. (21) method enabled to increase ice porosity by increasing the nucleation temperature and by near-isothermal ice growth. Despite different approaches exist to increase ice porosity, the method developed herein has the particularity of enabling localized nucleation at the bottom, by restraining the supercooled region to a relatively small volume layer. This approach can therefore improve consistency of the ice structure and thermal history between vials. This is a relevant difference from other methods that may cause nucleation at the top, such as ice fog (7) or pressure drop $(19,20)$, which in this case freeze in two stages, initially from top to bottom and after from bottom to top. Our approach, while falling into the group of other attempts to overcome the air gap caused by the curvature of the vial's bottom, provides a relatively simple standalone alternative.

Overall, the consistency of nucleation time and primary drying temperature profiles were significantly improved by

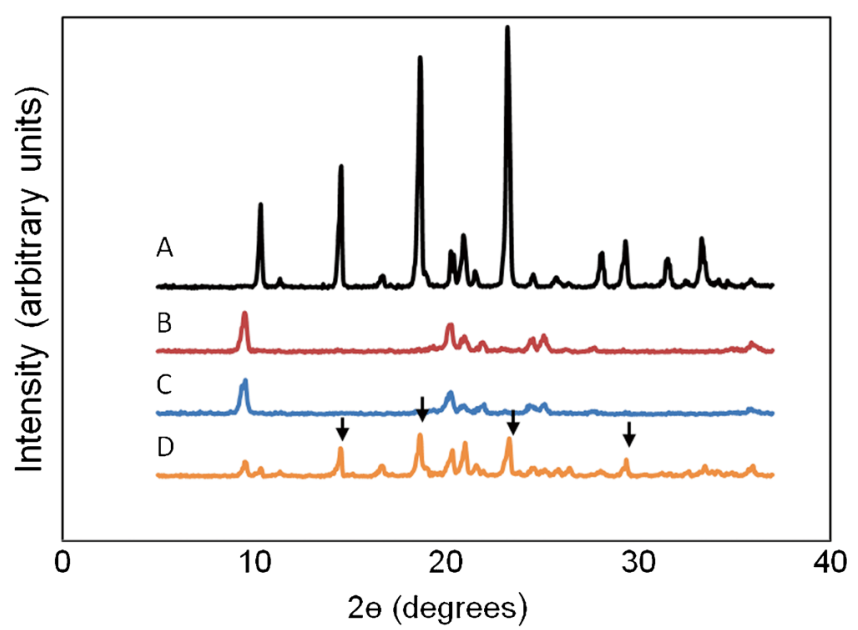

Fig. 10. PXRD diffractograms: a pure mannitol, b lyophilized powder from normal vial, c lyophilized powder from converted vial, and d lyophilized powder from converted vial which was radially frozen filling the bottom air gap with an adhesive material. Technical and regulatory limitations, not discussed herein, may pose relevant challenges to implement this approach to some industrial applications. That notwithstanding, this work also shows that many materials could provide crucial heattransfer enhancement under the principles discussed herein, which may enable sufficient design freedom to overcome the constraints.

\section{CONCLUSIONS}

The concave bottom of lyophilization vials causes a major heat-transfer limitation in lyophilization, which was addressed herein by rectifying the bottom surface of the vials with an adhesive material. This approach substantially enhanced the heat transfer, enabling consistent nucleation at the bottom of the vials and consequently unidirectional bottom to top freezing. The unidirectional ice structure formed was critical, enabling a $35 \%$ reduction of the primary drying time while preserving cake aspect under a more aggressive lyophilization cycle. When the ice structure was not unidirectional, the reduction of heat resistance at the bottom resulted in poor cake aspect. Overall, this work emphasizes the importance of unidirectional ice structure to generate low resistance honeycomb-structured cakes and how this could be achieved with a standalone approach $-\mathrm{a}$ vial with a rectified material at the bottom.

\section{ACKNOWLEDGMENTS}

This work was supported by Fundação para a Ciência e Tecnologia (Portugal) PTDC/EQU-EQU/104318/2008, PTDC/QUI-BIQ/119677/2010, SFRH/BD/79046/2011, and IF/01183/2013.

\section{REFERENCES}

1. Patel SM, Pikal MJ. Lyophilization process design space. J Pharm Sci. 2013;102(11):3883-7.

2. Brülls $\mathbf{M}$, Rasmuson $\mathbf{A}$. Heat transfer in vial lyophilization. Int $\mathbf{J}$ Pharm. 2002;246(1-2):1-16.

3. Rambhatla S, Pikal MJ. Heat and mass transfer scale-up issues during freeze-drying, I: atypical radiation and the edge vial effect. AAPS PharmSciTech. 2003;4(2):E14.

4. Pikal MJ, Roy ML, Shah S. Mass and heat transfer in vial freezedrying of pharmaceuticals: role of the vial. J Pharm Sci. 1984;73(9):1224-37.

5. Patel SD, Gupta B, Yalkowsky SH. Acceleration of heat transfer in vial freeze-drying of pharmaceuticals. I: Corrugated aluminum quilt. J Parenter Sci Technol. 1989;43(1):8-14.

6. Yalkowsky SH, Patel SD. Acceleration of heat transfer in vial freeze-drying of pharmaceuticals. II. A fluid cushion device. Pharm Res. 1992;9(6):753-8.

7. Rambhatla S, Ramot R, Bhugra C, Pikal MJ. Heat and mass transfer scale-up issues during freeze drying: II. Control and characterization of the degree of supercooling. AAPS PharmSciTech. 2004;5(4):e58.

8. Hottot A, Vessot S, Andrieua J. A direct characterization method of the ice morphology. Relationship between mean crystals size and primary drying times of freeze-drying processes. Dry Technol. 2004;22(8):2009-21.

9. Patapoff TW, Overcashier DE. The importance of freezing on lyophilization cycle development. BioPharm International. 2002;15(3). 
10. Rosa M, Lopes C, Melo EP, Singh SK, Geraldes V, Rodrigues MA. Measuring and modeling hemoglobin aggregation below the freezing temperature. J Phys Chem B. 2013;117(30):8939-46.

11. Miller MA, Rodrigues MA, Glass MA, Singh SK, Johnston KP, Maynard JA. Frozen-state storage stability of a monoclonal antibody: aggregation is impacted by freezing rate and solute distribution. J Pharm Sci. 2013.

12. Searles JA, Carpenter JF, Randolph TW. The ice nucleation temperature determines the primary drying rate of lyophilization for samples frozen on a temperature-controlled shelf. J Pharm Sci. 2001;90(7):860-71.

13. Shon M. The importance of controlling nucleation temperature during the freeze step. Introducing of controlyo nucleation ondemand technology on the new FTS/SP Scientific LyoStar 3 Freeze Dryer: SP Scientific.

14. Tang X, Pikal MJ. Design of freeze-drying processes for pharmaceuticals: practical advice. Pharm Res. 2004;21(2):191-200.

15. Roy ML, Pikal MJ. Process control in freeze drying: determination of the end point of sublimation drying by an electronic moisture sensor. J Parenter Sci Technol. 1989;43(2):60-6.

16. Hottot A, Vessot S, Andrieua J. Freeze drying of pharmaceuticals in vials: Influence of freezing protocol and sample configuration on ice morphology and freeze-dried cake texture. 2007;46(7):66674.

17. Kasper JC, Friess W. The freezing step in lyophilization: physico-chemical fundamentals, freezing methods and consequences on process performance and quality attributes of biopharmaceuticals. Eur J Pharm Biopharm. 2011;78(2):24863.
18. Hottot AN, Kyuya. Andrieua, Julien. Effect of ultrasoundcontrolled nucleation on structural and morphological properties of freeze-dried mannitol solutions. 2008;86(2):193-200.

19. Konstantinidis AK, Kuu W, Otten L, Nail SL, Sever RR. Controlled nucleation in freeze-drying: effects on pore size in the dried product layer, mass transfer resistance, and primary drying rate. J Pharm Sci. 2011;100(8):3453-70.

20. Geidobler R, Mannschedel S, Winter G. A new approach to achieve controlled ice nucleation of supercooled solutions during the freezing step in freeze-drying. J Pharm Sci. 2012;101(12):4409-13.

21. Kuu WY, Doty MJ, Rebbeck CL, Hurst WS, Cho YK. Gapfreezing approach for shortening the lyophilization cycle time of pharmaceutical formulations-demonstration of the concept. J Pharm Sci. 2013;102(8):2572-88.

22. Rodrigues MA, Balzan G, Rosa M, Gomes D, de Azevedo EG, Singh SK, et al. The importance of heat flow direction for reproducible and homogeneous freezing of bulk protein solutions. Biotechnol Prog. 2013;29(5):1212-21.

23. Izutsu K, Rimando A, Aoyagi N, Kojima S. Effect of sodium tetraborate (borax) on the thermal properties of frozen aqueous sugar and polyol solutions. Chem Pharm Bull (Tokyo). 2003;51(6):663-6.

24. OpenFOAM 2.3 User Manual, OpenFOAM Foundation, 2014.

25. Sandia National Labs KI, Los Alamos National Labs. Paraview: Parallel visualization application. 2000-2012. p. http:// paraview.org.

26. Kim AI, Akers MJ, Nail SL. The physical state of mannitol after freeze-drying: effects of mannitol concentration, freezing rate, and a noncrystallizing cosolute. J Pharm Sci. 1998;87(8):931-5. 